\title{
Percepção e registro de abuso de álcool e de doenças relacionadas num hospital geral universitário
}

\author{
A. A. Rosa, S.C. Gonçalves, S.D. Stefani, S. O. Martins, D. D. Rosa, A.Hunsche, R. M. Azeredo, L. H. \\ SCHÖNELL, M. M. RUSCHEL, S. G. S. DE BARROS \\ Trabal ho realizado no Hospital de Clínicas de Porto Alegre- Faculdade de Medicina da Universidade Federal do Rio Grande do Sul, \\ Porto Alegre, RS.
}

\begin{abstract}
RESUMO - Oвj etıvo. Avaliar a associação entre o resultado do teste CAGE aplicado em pacientes internados num hospital geral universitário (Hospital de Clínicas de Porto Alegre - HCPA) e a percepção e regístro de abuso de álcool e de doenças relacionadas pelas equipes de saúde.

Pacientes e Métodos. O delineamento envolveu três fases: a) aplicação do teste CAGE aos 385 pacientes adultos e adolescentes internados considerados elegíveis para o estudo; b) revisão dos prontuários dos pacientes CAGE-positivo e de um número igual de pacientes CAGE-negativo, estratificados por área de internação e sorteados, para identificar hábitos alcoólicos e a presença de doenças relacionadas ao uso de álcool; c) entrevista com os membros das equipes médicas responsáveis para avaliar sua percepção sobre o hábito alcoólico em seu paciente. As diferenças encontradas foram analisadas através do teste Qui-quadrado e do teste t de Student, com significância estatística em nível de 5\% bicaudal.

Resultados. Foram encontrados 51 pacientes CAGE-positivo (13\%). A equipe médica registrou
\end{abstract}

\section{INTRODUÇÃO}

A alta freqüência de problemas relacionados ao consumo excessivo de bebidas al coólicas, inclusive em nosso meio, salienta a importância da abordagem do alcoolismo, o qual, muitas vezes, não é diagnosticado por profissionais de saúde ${ }^{1}$. Um estudo recente encontrou prevalência de consumo excessivo de álcool em $13 \%$ da população masculina e em 3\% da feminina entre adultos residentes em Porto Alegre ${ }^{2}$. Outros trabalhos têm encontrado prevalências que variam entre $9 \%$ e $58 \%{ }^{3}$. Hospitais gerais e psiquiátricos, provavelmente, apresentam números maiores ${ }^{1,4}$.

A associação entre o consumo excessivo de ál cool e diversas patologias está demonstrada em várias séries na literatura ${ }^{5-9}$ sendo que estas enfermidades podem, em al guns casos, constituir-se no motivo principal da hospitalização.

E ntre os vários instrumentos disponíveis para a abuso de álcool no prontuário de $51 \%$ dos pacientes CAGE-positivo e $\mathbf{1 0 \%}$ dos CAGE-negativo, e a equipe de enfermagem fez este registro no prontuário de $31 \%$ dos pacientes CAGE-positivo e $8 \%$ dos CAGE -negativo $(p=724)$. Não houve diferença entre o registro e a percepção da equipe médica $(p=184)$. A prevalência de doenças relacionadas ao abuso de álcool foi $27 \%$ quando se examinou os prontuários médicos e $26 \%$ quando se entrevistou a equipe médica $(p=861)$, sendo maior entre os pacientes CAGE-positivo (43\%) do que entre os CAGE-negativo $(10 \%)(p=0003)$.

Conclusóns. 0 abuso de bebidas alcoólicas e a presença de doenças associadas ao mesmo são condições prevalentes nos pacientes admitidos ao HCPA. Entretanto, mais de metade dos casos não foram detectados pela equipe de saúde, havendo correspondência entre os registros de prontuário e a entrevista.

UnITERmos: Alcoolismo. Abuso de álcool. Doenças associadas.

detecção de bebedores abusivos e alcoólatras, ${ }^{10-14}$ existe o questionário CAGE. Este questionário foi testado, em 1974, por Mayfield et al. ${ }^{15}$ e consiste em quatro perguntas breves sobre o uso de bebidas al coólicas, com sensibilidade de $81 \%$ para duas ou mais questões respondidas afirmativamente. Masur e Monteiro' ${ }^{16}$ validaram sua versão em português, encontrando sensibilidade de $88 \%$ e especificidade de $83 \%$.

Com a intenção de estudar o consumo excessivo de bebidas alcoólicas entre os pacientes internados no Hospital de Clínicas de Porto Alegre (HCPA), delineou-se o presente estudo, o qual tem os seguintes objetivos específicos: 1) avaliar a prevalência de pacientes CAGE-positivo; 2) avaliar a preval ência de doenças rel acionadas ao consumo de ál cool; 3) determinar a associação entre a percepção na entrevista e o registro, no prontuário médico, a respeito do consumo excessivo de álcool; 4) determinar a associação entre o resultado do 
CAGE e o reconhecimento do problema pel as equipes médica e de enfermagem.

\section{PACIENTES E MÉTODOS}

Casos: todos os pacientes que responderam afirmativamente a duas ou mais perguntas do questionário CAGE (pacientes CAGE-positivo);

Não-casos: pacientes CAGE-negativo, estratificados por área de internação e sorteados em mesmo número que os CAGE-positivo;

Percepção: reconhecimento, pela equipe médica, do paciente como possuidor de problemas relacionados ao ál cool;

Registro: anotação da percepção no prontuário médico do paciente.

Este estudo foi realizado em todos os pacientes adolescentes e adultos hospitalizados nas áreas clínica, cirúrgica, obstétrica e psiquiátrica do HCPA. O trabalho foi desenvolvido em três fases: 1a) Aplicação do questionário CAGE, intercalado com outras onze perguntas sobre hábitos gerais para evitar vieses de aferição. Esta etapa foi realizada por cinco membros do grupo, previamente treinados em projeto piloto. O questionário era aplicado após consentimento verbal do paciente. Todos os pacientes CAGE-positivo foram considerados abusadores (casos). Após, foi sorteado número igual de pacientes CAGE-negativo (não-casos), procedentes da mesma área de internação. 2a) Três membros do grupo, não envol vidos na apl icação do questi onário, fizeram a revi são dos prontuários dos componentes das duas amostras (casos e nãocasos), para a obtenção da prevalência das doenças relacionadas ao consumo de álcool. 3a) Foi feita a redistribuição dos pacientes das duas amostras aos entrevistadores, de modo a manter o cegamento na etapa seguinte.Logo após, realizou-se a entrevista padronizada, aplicada aos membros das equipes médicas responsáveis pelos pacientes das duas amostras (amostragem não-probabilística consecutiva de todos os pacientes CAGE-positivo; amostragem aleatória estratificada de pacientes CAGE-negativo, pareados em mesmo número e por local de origem, com os CAGE-positivo).

Foram estudadas as seguintes variáveis: resultado do teste CAGE, diagnóstico de doença relacionada à ingestão de álcool, percepção por parte do membro da equipe médica responsável (na entrevista), sexo, idade, raça, grau de escolaridade, tempo de internação, registro do problema, no prontuário, pel a equipe médica e pela equipe de enfermagem.

F oram excluídos os pacientes submetidos a procedimentos cirúrgicos no dia da aplicação do questionário, os não passíveis de recuperação pela
Tabela 1 - Entrevista com a equipe médica: percepção da equipe médica sobre abuso de álcool pelos pacientes.

\begin{tabular}{|lcccc|}
\hline $\begin{array}{c}\text { Abuso de } \\
\text { Álcool }\end{array}$ & $\begin{array}{c}\text { Cage- } \\
\text { Positivo }\end{array}$ & $\begin{array}{c}\text { Cage- } \\
\text { Negativo }\end{array}$ & Total & $\begin{array}{c}\% \\
\text { Cumulativo }\end{array}$ \\
Sim & $26(51 \%)$ & $5(10 \%)$ & $31(30 \%)$ & 30 \\
Não & $19(37 \%)$ & $40(78 \%)$ & $59(58 \%)$ & 88 \\
Ignorado & $6(12 \%)$ & $6(12 \%)$ & $12(12 \%)$ & 100 \\
Total & 51 & 51 & 102 & - \\
\hline
\end{tabular}

equi pe de campo, os cl ini camente impossibilitados de responder e aqueles não encontrados no leito entre 8 e 20 horas. O protocolo deste trabal ho foi avaliado e aprovado pel o Grupo de Pesquisa e PósGraduação e pela Comissão de É tica do Hospital de Clínicas de Porto Alegre.

A análise estatística foi realizada utilizando-se o programa de computação E pi-I nfo 5.01b. Os testes utilizados foram o Qui-quadrado, na comparação entre variávei s qual itativas, e o testet de Student, na comparação entre variáveis quantitativas, com significância estatística em nível de 5\% bicaudal.

\section{RESULTADOS}

Dos 430 pacientes internados, foram entrevistados 385 (89,5\%), sendo 195 (51\%) do sexo feminino. A média de idade foi de 48 anos, sendo que 323 pacientes (84\%) eram brancos. O nível de escolaridade predominante foi o primei ro grau incompleto (50\%); 29 pacientes ( $8 \%$ ) eram analfabetos e um mesmo número possuía curso superior completo.

A maioria dos pacientes pertencia à área clínica (50\%), seguida da cirúrgica (30\%), gineco-obstétrica $(12 \%)$ e psiquiátrica (8\%). A prevalência de teste CAGE-positivo no Hospital de Clínicas de Porto Alegre (HCPA) foi de 13\% (51 pacientes).

$\mathrm{Na}$ entrevista com a equipe médica, obteve-se informação sobre o consumo de ál cool em $88 \%$ dos pacientes. Foram considerados abusadores 51\% dos pacientes CAGE-positivo e $10 \%$ dos CAGEnegativo. A equipe médica não soube informar sobre o consumo de álcool em $12 \%$ dos pacientes CAGE-positivo e no mesmo percentual de CAGE negativo. A tabela 1 sumariza esses resultados.

A tabela 2 demonstra que $70 \%$ dos prontuários pesquisados mencionavam dados a respeito de abuso de álcool - resultado comparável ao da entrevista $(p=.184)$. Dos pacientes CAGE-positivo, $51 \%$ foram considerados abusadores e não havia menção sobre uso de ál cool em $26 \%$ deles. Entre os pacientes CAGE-negativo, $10 \%$ foram classificados como abusadores e não havia menção de álcool em $33 \%$ deles. 
Tabela 2 - Pesquisa dos prontuários: registro da equipe médica sobre abuso de álcool pelos pacientes.

\begin{tabular}{|lcccc|}
\hline $\begin{array}{c}\text { Abuso de } \\
\text { Álcool }\end{array}$ & $\begin{array}{c}\text { Cage- } \\
\text { Positivo }\end{array}$ & $\begin{array}{c}\text { Cage- } \\
\text { Negativo }\end{array}$ & Total & $\begin{array}{c}\% \\
\text { Cumulativo }\end{array}$ \\
Sim & $26(51 \%)$ & $5(10 \%)$ & $31(30 \%)$ & 30 \\
Não & $12(24 \%)$ & $29(57 \%)$ & $41(40 \%)$ & 70 \\
Ignorado & $13(26 \%)$ & $17(33 \%)$ & $30(30 \%)$ & 100 \\
Total & 51 & 51 & 102 & - \\
\hline
\end{tabular}

Tabela 3 - Pesquisa dos prontuários: registro da equipe de enfermagem sobre abuso de álcool pelos pacientes.

\begin{tabular}{|lcccc|}
\hline $\begin{array}{c}\text { Abuso de } \\
\text { Álcool }\end{array}$ & $\begin{array}{c}\text { Cage- } \\
\text { Positivo }\end{array}$ & $\begin{array}{c}\text { Cage- } \\
\text { Negativo }\end{array}$ & Total & $\begin{array}{c}\% \\
\text { Cumulativo }\end{array}$ \\
Sim & $16(31 \%)$ & $4(8 \%)$ & $20(20 \%)$ & 20 \\
Não & $25(49 \%)$ & $37(73 \%)$ & $62(61 \%)$ & 80 \\
Ignorado & $10(20 \%)$ & $10(20 \%)$ & $20(20 \%)$ & 100 \\
Total & 51 & 51 & 102 & - \\
\hline
\end{tabular}

A equipe de enfermagem fez menção ao uso de álcool em $80 \%$ do total dos prontuários (tabela 3), resultado comparável ao da equipe médica $(p=142)$. Dos pacientes CAGE-positivo, 31\% foram considerados abusadores e não havia menção sobre este hábito em $20 \%$ deles. Dos pacientes CAGE-negativo, $8 \%$ foram classificados como abusadores e em $20 \%$ não havia menção sobre uso de álcool. Comparando-se os registros sobre abuso de álcool, não houve diferença estatisticamente significativa entre a equipe de enfermagem e a equipe médica $(p=.724)$.

$\mathrm{Na}$ entrevista com a equipe médica (tabela 4), $39 \%$ dos CA GE-positivo e $12 \%$ dos CAGE-negativo foram considerados possuidores de doenças relacionadas ao consumo excessivo de álcool $(p=0,003)$.

Na revisão dos prontuários (tabela 5), verificou-se que $43 \%$ dos CAGE-positivo e $10 \%$ dos CAGEnegativo apresentavam doenças relacionadas ao uso de álcool $(p=0,0003)$. E ssas prevalências foram semel hantes àquelas obtidas através da entrevista com a equipe médica $(p=.861)$.

\section{DISCUSSÃO}

Os resultados deste estudo mostram uma prevalência maior (13\%) de provável consumo excessivo de bebidas alcoólicas do que a encontrada na população geral de nosso meio, em torno de $6 \%$, quando não estratificada por sexo².

Em relação aos pacientes considerados abusadores de álcool, houve concordância entre a entrevista com a equipe médica e os registros nos prontuários. Permite-se supor, então, que a falha na
Tabela 4 - Entrevista com a equipe médica: freqüência de doenças relacionadas ao uso de álcool (DRA) entre pacientes CAGE-positivo e CAGE-negativo.

\begin{tabular}{|lcccc|}
\hline DRA & $\begin{array}{c}\text { Cage- } \\
\text { Positivo }\end{array}$ & $\begin{array}{c}\text { Cage- } \\
\text { Negativo }\end{array}$ & Total & $\begin{array}{c}\% \\
\text { Cumulativo }\end{array}$ \\
Presente & $20(39 \%)$ & $6(12 \%)$ & $26(26 \%)$ & 26 \\
Ausente & $31(61 \%)$ & $45(88 \%)$ & $76(75 \%)$ & 100 \\
Total & 51 & 51 & 102 & - \\
\hline
\end{tabular}

\begin{tabular}{|c|c|c|c|c|}
\hline DRA & $\begin{array}{l}\text { Cage- } \\
\text { Positivo }\end{array}$ & $\begin{array}{c}\text { Cage- } \\
\text { Negativo }\end{array}$ & Total & $\begin{array}{c}\% \\
\text { Cumulativo }\end{array}$ \\
\hline Presente & 22 (43\%) & $5(10 \%)$ & 27 (27\%) & 27 \\
\hline Ausente & 29 (57\%) & $46(90 \%)$ & 75 (74\%) & 100 \\
\hline Total & 51 & 51 & 102 & - \\
\hline
\end{tabular}

detecção de possíveis pacientes com problemas relacionados ao álcool ocorra devido à falta de investigação e diagnóstico, e não apenas aos registros incompletos.

$\mathrm{Na}$ entrevista, a equipe médica mencionou que 59 pacientes, do subtotal da amostra estudada, não eram considerados abusadores, mas registrou tal informação somente em 41 prontuários. Em 30 prontuários, onde não havia qualquer menção sobre álcool por parte da equipe médica, 13 pertenciam aos pacientes considerados abusadores (CAGE-positivos). Esses achados evidenciam que houve importante falta de registro.

A equipe de enfermagem deixou de registrar informação a respei to do uso de ál cool numa quantidade menor de prontuários do que a equipe médica, embora tal diferença não tenha sido estatisticamente significativa. Talvez, devido ao fato de existirem questionários padronizados, preenchidos pela equipe de enfermagem no momento da admissão de cada paciente, entre os quais há uma pergunta sobre uso de bebidas alcoólicas. Já no caso da equi pe médica, que não utiliza questionários para seus pacientes no momento da anamnese, a investi gação do hábito de beber depende da importância que cada profissional atribui ao alcoolismo. Provavelmente, a abordagem do alcoolismo varia de acordo com a especialidade de cada médico, sendo que estudos posteriores para a confirmação desta hipótese são necessários.

A equi pe médi ca consi der ou como abusadores de álcool metade dos pacientes CAGE-positivo, enquanto a equipe de enfermagem, apenas um terço deles. Isso denota a necessidade de uma melhor 
abordagem pela equipe de saúde, em relação à ingestão de bebi das alcóolicas.

E $m$ relação à presença de doenças associadas ao uso abusi vo de álcool, houve al guma concor dância entre os achados na entrevista com a equi pe médica (percepção) e os registros nos prontuários, estando as mesmas presentes em 26 e $27 \%$ dos pacientes do total da subamostra estudada, respectivamente. Estes dados superam os encontrados na literatura, que se situam ao redor de $5 \%{ }^{17}$. Houve maior prevalência de doenças relacionadas ao uso de álcool nos pacientes CAGE-positivo, quando comparados aos CAGE-negativo, o que mostra a utilidade do questionário CAGE como instrumento de triagem de possíveis abusadores, os quais têm grandes possibilidades de apresentar problemas decorrentes dessa ingesta excessiva.

Os profissionais de saúde, freqüentemente, não reconhecem a associação entre problemas clínicos comuns e o uso excessivo de bebidas alcoólicas, sendo constante o tratamento das complicações orgânicas relacionadas ao álcool sem a abordagem do problema básico, que é a ingestão alcoólica. O al cool ismo precisa ser visto como uma doença crônica, passível de ser controlada quando estabelecido o adequado tratamento, da mesma forma que a hipertensão arterial sistêmica ou o diabete mélito ${ }^{18}$. Nosso estudo mostrou que ainda há faIhas na percepção e no registro pelas equipes médica e de enfermagem, de pacientes que, provavelmente, consomem álcool em excesso.

Conclui-se que, apesar de largamente descrito na literatura como problema de saúde, o alcoolismo não está sendo adequadamente investigado e, conseqüentemente, registrado. N ossos resultados, portanto, corroboram os dados da literatura quanto à precariedade dos sistemas de registro e do reconhecimento dos casos pela equipe de saúde ${ }^{17}$. O questionário CAGE é um instrumento simples que pode auxiliar uma anamnese bem feita, uma vez que possui boa sensibilidade e especificidade (88\% e $83 \%$, respectivamente $)^{16}$. Além de sua utilidade como método simpl es de rastreamento, el e guarda também correlação com a presença de doenças relacionadas ao ál cool. Embora ainda não exista consenso quanto à interpretação do verdadeiro valor de um teste "CAGE-positivo", sabe-se que este resultado se associa ao consumo excessi vo de bebidas al coólicas. Dessa forma, pacientes que preenchem esse critério devem ser adequadamente avaliados em relação à presença de doenças rel acionadas ao álcool. Para que isto ocorra, é necessário que a equipe de saúde não subestime o uso de álcool como fator de risco e o investigue e registre rotineiramente.

\section{SUMMARY}

\section{Perception and registration of alcohol abu- se and alcohol related diseases by nurses and medical staff of an university general hospital}

BACKGROUND. The high frequency of alcohol re lated problems highlights the importance of its approach. The association of both the alcohol-abuse and alcohol related diseases has been pointed in the literature and are often the main reason for hospitalization. Physicians use different tools to detect abusive drinkers, and one of them is the CAGE-test, validated by Mayfield et al. in 1974.

OBJ ECTIVES. To study the association between the result of the CAGE-test and the perception and registration of alcohol abuse by the medical staff and the nurses in charge of inpatients of Hospital de Clínicas de Porto Alegre, southern Brazil.

Material And Method. The design involved three phases. First, the application of the CAGEtest to the 385 eligible patients. Second, review of all 51 CAGE-positive patients' records as well the controlled evaluation of 51 randomized CAGEnegative patients, to seek alcoholic habits and alcohol-related diseases. Third, interview with the medical staff to find out their perception about these subjects.

RESULTS. We found 51 patients CAGE-positive (prevalence $=13 \%$ ). When evaluated through the registration and through a standardized interview, medical staff and nurses were not aware of more than half of CAGE-positive patients. No significant differences were found between medical staff registration and perception; the prevalence of alcohol related diseases was the same for both: 26 and $27 \%(p=.861)$. The prevalence of alcohol related diseases was higher among CAGE-positive patients ( $p=.0003$ ).

Conclusions. In spite of the high prevalence among our patients, alcohol abuse is not pursued by our health teams as it should be. As for the future, there is reason to envision a continuing alcoholism rapprochement. [Rev Ass Med Brasil 1998; 44(4): 335-9.]

KEY WORDS: Alcoholism. Alcohol abuse. Alcohol related diseases.

\section{REFERÊ NCIAS BIBLIOGRÁFICAS}

1. Hasin DS, Grant BF, Dufour MG, Endicott J . Alcohol problems increase while physician attention declines. Arch Intern Med 1990; 150: 397-400. 
2. Duncan B. As desigualdades sociais na distribuição defatores de risco para doenças não transmissíveis (Tese, Doutorado). Porto Alegre: Universidade Federal do Rio Grande do Sul, 1991. $216 \mathrm{p}$.

3. Soil beman M, Luz J r E. Problemas relacionados ao consumo de álcool. In: Duncan B, Schmidt M \& Giugliani E (Ed.). Medicina Ambulatorial. Porto Alegre, Artes Médicas, 1990. p.191-7.

4. Capriglione MJ, Monteiro MG,Masur J. Aplicação do Questionário CAGE para detecção da síndrome de dependência do álcool em 700 adultos na cidade de São Paulo. Rev Ass Bras Psiq 1985; 7: 50-3.

5. Thomas DB. Bladder cancer and alcoholic beverage comsuption. AmJ Epidemiol 1983; 118: 720-7.

6. Pollak E. Prospectivestudy of al cohol comsuption and cancer. N Engl J Med 1984; 310: 617-21.

7. Kellman PA. Alcohol comsuption and diabetes mellitus mortality in different countries. Am J Publ Health 1983; 73: 1.316-7.

8. Masur J . Hipertensão e al coolismo. Rev Ass Med Brasil 1980; 26: 86-8.

9. Vianna LC, Monteiro MG. Utilidade do questionário CAGE para identificação de problemas relacionados ao álcool entre as causas de mortalidade de mulheres resi dentes em Diadema (SP) no período de 1985-1986. Rev Ass Med Brasil 1991; 37: 139-42.
10. National Institute on Alcohol Abuse and Alcoholism. NIAAA issues report on screening for al coholism. AFP 1990; 42: 1664-6.

11. Editorial. Screening tests for alcoholism. Lancet 1988; 11: 1.117-8.

12. Cyr MG, Wartman S. The effectiveness of routine screening questions in detection of alcoholism. J AMA 1988; 259: 51-4.

13. J orge MR. O diagnóstico do alcoolismo: entrevistas estruturadas, questionários eexames delaboratório. In: Ramos SP (E d.). Alcoolismo hoje. Porto Alegre, Artes Médicas, 1987. p. 50-9.

14. Selzer ML. The Michigan Alcoholism Screening Test: the quest for a new diagnosis instrument. AmJ Psychiatry 1971; 127: 1.653-8.

15. Mayfeld D, McLeod MS, Hall P. The CAGE Questionnaire: validation of new alcoholism screening instrument. Am J Psychiatry 1974; 131: 1.121-3.

16. Masur J, Monteiro MG. Validation of the "CAGE" al coholism screening test in Brazilian psychiatric inpatient hospital setting. BrazJ Med Biol Res 1983; 16: 215-8.

17. Santana VS, Almeida Filho N. Aspectos epidemiológicos do alcoolismo. In: Ramos SP (Ed.). Alcool ismo hoje, Porto Alegre. Artes Médicas, 1996. pp. 36-54.

18. Barros S, Galperin B. Problemas clínicos comuns do alcoolista. In: Ramos SP (Ed.). Alcoolismo hoje. Porto Alegre, Artes Médicas, 1987. p. 75-99. 